\title{
"YOU BETTER WERK". RASGOS DEL CAMP TALK EN LA SUBTITULACIÓN AL ESPAÑOL DE RUPAUL'S DRAG RACE
}

\author{
Iván Alejandro Villanueva Jordán ${ }^{1}$ \\ ${ }^{1}$ Universidad Peruana de Ciencias Aplicadas, Monterrico, Santiago de Surco,
}

Peru

\begin{abstract}
Resumen: Este artículo aborda la subtitulación en castellano del programa de televisión de realidad RuPaul's Drag Race (RPDR) mediante un análisis porcentual de la presencia de rasgos microtextuales relativos al camp y la manera en que estos fueron traducidos en las versiones publicadas en Netflix y en el grupo de Facebook RPDR Venezuela. El artículo inicia con una elaboración sobre el camp (una sensibilidad estética comúnmente asociada con la identidad gay y difundida en espacios anglófonos) y el camp talk como categorías de estudio para la traductología. Más adelante se presentan los resultados del análisis de los subtítulos de dos episodios del programa de televisión. Finalmente, la discusión sobre los resultados alcances sobre el papel de la traducción en los procesos de aculturación relacionados al camp, así como sobre las nuevas funciones sociales de la subtitulación.
\end{abstract}

Palabras clave: Traducción Audiovisual; Drag Queen; Subtitulación; Camp; RuPaul's Drag Race

\section{"YOU BETTER WERK". CAMP TALK FEATURES IN THE SPANISH SUBTITLES OF RUPAUL'S DRAG RACE}

\begin{abstract}
This article addresses Spanish subtitling for the reality television show RuPaul's Drag Race (RPDR), which has been aired in the United States since 2009, analyzing the presence of specific microtextual features of camp (an aesthetic sensibility present in English-speaking spaces that is generally associated with homosexual identity) and of the manner in which
\end{abstract}


these features have been translated into the Spanish subtitled versions found on Netflix and produced by the Facebook group RuPaul's Drag Race Venezuela. The article begins with a description of the importance of researching camp talk in the context of Translation Studies and continues with an analysis of subtitles from two of the show's episodes. Finally, results are discussed concerning the scope of translation's role in camprelated acculturation processes, and new social functions for subtitling are considered.

Keywords: Audiovisual Translation; Drag Queen; Subtitling; Camp; RuPaul's Drag Race

\section{Introducción}

Hasta hace un par de décadas, la palabra drag queen no resistía el tamiz lingüístico del castellano y la subtitulación de algunas películas ahora ya clásicas -como The Adventures of Priscilla, Queen of The Desert (Elliot), To Wong Foo. Thanks for Everything, Julie Newmar (Kidron) o The Birdcage (Nichols) - proponía como equivalentes palabras como travesti, transformista, reinona, entre otras. Desde entonces hasta ahora, la imagen de las drag queens y el dragqueenismo — el conjunto de actividades y saberes que implica performar como una drag queen (Villanueva Jordán 98) - se han hecho mundiales y coexisten con distintas prácticas e identidades alternas a la heteronormatividad — «the cultural expectation that people not only adhere to the gender order, but that they also naturally desire the "opposite" sex»(Jones 211). Este fenómeno de mundialización del dragqueenismo, en principio estadounidense, trae consigo no solo el imaginario, sino también el hecho lingüístico de esta forma de performar: el camp talk. De esta forma, una palabra como condragulations o expresiones como Sashay-away o Shantay, you stay tienen destinos diferenciados en la subtitulación comercial del programa de televisión de realidad RuPaul's Drag Race (RPDR) y aquella realizada por los aficionados.

La forma de hablar camp no es nueva para la traductología; el antecedente directo que asumo de este breve trabajo es la 
investigación de Harvey sobre el uso del camp en la literatura gay estadounidense de la década de 1970, cómo este uso se tradujo al francés y las implicancias que tal transferencia lingüística tuvo a nivel comunitario e identitario en el sistema receptor. El estudio de Harvey continúa siendo una referencia importante para las investigaciones que vinculan los Estudios Culturales con la traducción, dado que amplía el debate de las relaciones de género más allá de la categoría mujer. Otro influjo en el estudio del camp talk, aunque centrado más bien en una conceptualización más lingüística como es el gayspeak, proviene de la investigación de Ranzato que analiza el doblaje del inglés al italiano y las representaciones de sujetos gay en algunos productos audiovisuales de consumo masivo.

Tanto el trabajo de Harvey como el de Ranzato plantean conclusiones interesantes sobre el rol de la traducción como una herramienta de diagnóstico del cambio social, incluso como la propia herramienta de cambio. En el caso de Harvey ("Gay Community, Gay Identity and the Translated Text" 156), la exportación de modelos identitarios gais de Estados Unidos a Francia, visibles inclusive en la manera de hablar de los personajes (el camp talk), se vio detenida por la manera en que la comunidad homosexual aún no tenía bases conceptuales, léxicas o incluso comunitarias en el país receptor. De la misma forma, Ranzato (382) plantea que la carga semántica de muchos equivalentes utilizados en el doblaje italiano del gayspeak se centran solo en antiguos estereotipos de la homosexualidad y no reflejan los matices o los aspectos semióticos de las expresiones actuales.

Este trabajo retoma el camp talk (y lo diferencia del gayspeak) para estudiar su traducción en los medios audiovisuales, en particular como producto de una modalidad específica de la traducción audiovisual, la subtitulación. Mediante un análisis contrastivo de las representaciones camp en los diálogos en inglés de RPDR y dos tipos de versiones subtituladas al castellano (una comercial y otra hecha por aficionados), se busca identificar la manera en que la traducción del camp talk neutraliza o reproduce de alguna 
manera dichas representaciones. Asimismo, de forma transversal a este breve estudio, se propondrán ideas para comprender la subtitulación como una práctica social que ya ha sobrepasado la esfera profesional de la traducción y que ahora cumple funciones sociales diversas, entre ellas algunas identificables en los subtítulos y la subtitulación realizada por aficionados.

\section{Representaciones camp y el camp talk}

Las palabras del inglés camp (sust.), to camp (v.) o campy (adj.) parecen provenir del francés se camper -el uso de estas palabras en contexto puede encontrarse en la etnografía de Paulson y Simpson-. Booth se refiere a la definición de camp del diccionario Passing English of the Victorian Era como «actions and gestures of exaggerated emphasis». Si bien el componente de la exageración se mantiene aún en la palabra camp, esta ahora abarca también el afeminamiento y, por algunas extensiones de esta idea, la homosexualidad: "Robin was totally insane. He had a voice like an air-raid siren. He was campy and didn't care what people thought [...]» (Paulson and Simpson 37). Sin embargo, con el desarrollo de los estudios identitarios gais, el concepto de camp ha adquirido una perspectiva crítica, vinculada principalmente con la estética y la política. La perspectiva estética puede rastrearse hasta la diferencia que hace Christopher Isherwood sobre high camp y low camp (Bergman) o hasta el ensayo de 1964 de Susan Sontag «Notes on Camp» vapuleado por distintos teóricos de la crítica gay.

Camp is as well a quality discoverable in objects and the behavior of persons. There are "campy" movies, clothes, furniture, popular songs, novels, people, buildings... This distinction is important. True, the Camp eye has the power to transform experience. But not everything can be seen as Camp. It's not all in the eye of the beholder. (Sontag 277) 
La perspectiva estética identificable en el ensayo de Sontag se relaciona con el vínculo entre los objetos y los sujetos que reconocen en estos objetos características campy. Este intercambio no se produce porque los objetos tengan características intrínsecas del camp, sino porque los espectadores interpretan de esta manera lo que ven, a partir de experiencias previas o conocimientos culturales que les permiten proyectar estos significados en los objetos o crearlos mediante su propia manera de actuar; estas ideas se relacionan con el primer sistema de representación que, según Stuart Hall, permite

give meaning to the world by constructing a set of correspondences or chain of equivalences between things -people, objects, events, abstract ideas, etc. - and our system of concepts, or conceptual maps" (Hall 17)

Por otro lado, Sontag menciona también la vinculación entre la homosexualidad y el camp, aunque sin desarrollar la dinámica o razón de ser de tal vínculo - lo que tal vez haya terminado por hacer que los críticos la culparan por la muerte de un concepto que pudo haber sido reivindicado debido a sus verdaderas funciones sociales y no por lo que, en la superficie, era una cuestión de gustos-. Con el tiempo surgieron nuevas interpretaciones del vínculo entre el camp y la homosexualidad que, aunque también partían de la perspectiva estética, revelaban su faceta política. Entre estas interpretaciones se puede mencionar el fecundo trabajo etnográfico de Esther Newton sobre las drag queens en Estados Unidos, Mother Camp (1972).

Esta antropóloga realiza una comparación entre la relación del soul con la comunidad afroamericana y el camp con la comunidad homosexual (Newton 105); en ambos casos, arguye, se trataría de una sensibilidad desarrollada sobre la base de experiencias compartidas por los sujetos que asumen una identidad particular (afroamericana o gay). Estos procesos de aculturación pueden 
suceder en el sujeto cuando este decide resistirse a experimentar el mundo de acuerdo a la heteronormatividad y comienza a "acquire a conscious identity, a common culture, a particular outlook on the world, a shared sense of self, an awareness of belonging to a specific social group, and a distinctive sensibility or subjectivity" (Halperin 6). Dicha sensibilidad buscaría no solo producir respuestas estéticas frente a determinadas prácticas o elementos culturales compartidos, sino también responder frente a situaciones (de tensión o amenaza de los otros, o de coloquialidad entre pares).

La conceptualización del camp como sensibilidad estética o práctica política es un tema de gran importancia para los estudios gais y puede extenderse al análisis de distintos productos culturales (cine, televisión, música, moda, etc.) en el marco de lo que los Estudios Culturales han denominado el circuito cultural o incluso desde perspectivas semióticas. Sin embargo, para fines de este estudio, es necesario un enfoque solo en la configuración lingüística del camp y comprenderla como una forma de comunicación entre pares, de uso comunitario, que viene construyéndose precisamente por experiencias compartidas o por la creación de tradiciones culturales restringidas, por lo general, a espacios anglófonos.

\subsection{Estudios lingüísticos del gayspeak y el camp talk}

Parto de la idea de que el gayspeak, como concepto, ha tenido un desarrollo controversial en el marco de los estudios lingüísticos; ello se debe a que las primeras descripciones de este uso de la lengua (en particular, el léxico) estuvieron vinculadas con una mirada patologizante, moralista y condenatoria con respecto a los propios sujetos homosexuales. Un trabajo de estas características es, sin duda, el de Legman (publicado en 1941) The Language of Homosexuality. An American Glossary. Cameron and Kulick proponen que esta «primera» etapa tuvo lugar en el periodo 1920-1940 y lo que vino luego, entre 1950 y 1960, fue una propuesta reivindicadora del uso de la lengua como un instrumento de afirmación social y política: a nivel identitario, la palabra gay se impuso como la etiqueta 
representativa de toda una comunidad. En efecto, las acciones afirmativas de dicho grupo que intentaban marginalizar otras formas de expresión homosexual (como la de las drag queens y el camp en general) tuvieron éxito en los años siguientes.

Una tercera etapa en el desarrollo de la sociolingüística gay vendría en la década del setenta y se extendería hasta mediados de los noventa con el (re)surgimiento de los enfoques descriptivos y la acuñación propiamente dicha del término gayspeak (Cameron and Kulick 87-98; Hayes). Aquí resulta necesario plantear que el concepto del gayspeak no era uniforme y variaba de acuerdo al enfoque de los investigadores y el alcance de sus estudios (Gray 229); sin embargo, se puede hacer una conceptualización general al decir que el gayspeak implicaba la manera en que los sujetos homosexuales, que se identificaban a sí mismos como gais, utilizaban la lengua. Los alcances de este tipo de estudios estaban vinculados con el léxico (los insultos, la jerga, la feminización), las circunstancias en las que esta forma de hablar se movilizaba y la manera en que estos usos de la lengua podían servir para identificar los estratos o grupos sociales dentro de esta comunidad o también llamada subcultura gay. Si bien estas propuestas ya habían dejado de lado la perspectiva moralizante de décadas atrás, avanzaban una idea homogeneizadora sobre lo que significaba no ser heterosexual: es decir, ser gay (o lesbiana). En cierta medida, esta mirada académica promovía una oposición binaria que elidía otras identidades divergentes, así como sus formas de hablar.

Estudiar el camp como una manera de hablar se diferencia de las propuestas enfocadas en el gayspeak por distintas razones. El gayspeak, como categoría de estudio, puede ser más amplio en su alcance, al abarcar desde la fonética (por ejemplo, el denominado gay lisp de la pronunciación de las sibilantes en inglés), pasando por el léxico (el polari como jerga), lo semántico (el uso de metáforas para codificar el deseo), la sintaxis, hasta lo suprasegmental (la identificación del tono irónico). Asimismo, como evidencia su denominación, existe una vinculación con la identidad gay que hace que distintas variables, como las dialectales, entren en 
juego al momento de realizar la selección de la población y las muestras. Parece que los métodos recurrentes en los estudios del gayspeak suelen ser de la lingüística descriptiva (enfocados en la formalización de los usos descritos). Como se mencionará más adelante, las categorías de identificación y análisis del camp tienen una profunda relación con las dimensiones semiótica y pragmática de la lengua: los referentes culturales, la intertextualidad, las implicaturas, las presuposiciones, entre otros.

\subsection{Problemáticas de la identificación del camp}

Con el surgimiento de la teoría queer, se produce una caída de las identidades unificadas por los movimientos gais de mayor impacto político en la década de 1990. Esto permite, a su vez, que otras expresiones identitarias se pongan de relieve como modelos alternativos en contra de la heteronormatividad. De esta manera, las masculinidades periféricas, en particular las de las campy queens (hombres gais afeminados), comienzan a ser parte de las revalorizaciones identitarias y también del foco de estudio de disciplinas vinculadas con temas sociales y las humanidades, como la sociología, la historia, la antropología, las ciencias de la comunicación y la crítica literaria. El camp se convierte entonces en un tema o subtema de estudio abordado repetidamente en su expresión superestructural (como discurso, como estética, como práctica política). Sin embargo, no todas estas propuestas han sido satisfactorias en la descripción clara de cómo se constituye.

Entre las propuestas más congruentes en la conceptualización del camp, se encuentra la David Halperin en su libro How to Be Gay. Si bien la teorización sobre el camp es amplísima y cuenta con distintos enfoques, el trabajo de este autor es una propuesta crítica sobre las formas en que el camp se utiliza como una estética comunitaria con fines de identificación y defensa. Se trata de un trabajo que articula aportaciones anteriores sobre el tema y plantea pistas para la identificación de patrones camp en distintas representaciones de los medios de comunicación. Halperin sostiene que el camp tiene 
expresiones de alcance global que derivan del proceso de aculturación de los grupos sexuales minoritarios alrededor del mundo.

Las características que he decidido extraer de la propuesta de Halperin (206-20) son dos lo suficientemente amplias para luego abordar evidencias particulares: la identificación con la feminidad y la hilaridad de la feminidad. Se trata de una postura ambigua y estratégica con respecto los modelos de feminidad comúnmente relacionados con los sujetos gais. Según esta postura, estos sujetos se identifican con la posición femenina culturalmente construida de la mujer y la manera en que esta posición hace de ella un sujeto pasivo de situaciones dramáticas, dolorosas y, al mismo tiempo, cómicas. Esto significa que el camp vincula al sujeto gay con los modelos de feminidad y el perjuicio que estos representan potencialmente para el sujeto. Sin embargo, ante cualquier posibilidad de interpelación negativa, el sujeto gay se ríe, toma distancia de la interpelación y asume su vinculación con la feminidad; se trata de la aplicación de un esencialismo con fines estratégicos. "Camp works to drain suffering of the pain that it also does not deny" (Halperin 186).

Al volver sobre el trabajo de Harvey (2000A; 2000B; 2000C; 2002) se puede rastrear estas dos características del camp - la identificación y la desidentificación-particularizadas en estrategias y rasgos superficiales del uso de la lengua para poder identificarlas en la narrativa gay de la década de 1970. Como se ve en la tabla, en el caso de las estrategias (paradoja, hilaridad, parodia), Harvey plantea que son posiciones retóricas que permiten que los hablantes modulen las potencialidades de la lengua de acuerdo a sus intenciones y al contexto (Harvey, "Describing Camp Talk: Language/pragmatics/politics” 243).

Tabla 1 - Propuesta de descripción del camp talk de Harvey ("Describing Camp Talk: Language/pragmatics/ politics”)

\begin{tabular}{l|l}
\hline Estrategia & Características superficiales \\
\hline Paradoja & $\begin{array}{l}\text { mediante las incongruencias del registro, entre lo } \\
\text { explícito y lo implícito, entre la alta cultura y la } \\
\text { baja cultura. }\end{array}$ \\
\hline
\end{tabular}

Cad. Trad., Florianópolis, v. 39, no 3, p. 156-188, set-dez, 2019. 


\begin{tabular}{l|l}
\hline Inversión & $\begin{array}{l}\text { de los nombres propios marcados por el género, } \\
\text { los marcadores gramaticales del género, las } \\
\text { rutinas retóricas esperadas, los sistemas de valores } \\
\text { establecidos }\end{array}$ \\
\hline Hilaridad & $\begin{array}{l}\text { mediante formas de denominación motivadas, } \\
\text { juegos de palabras, dobles sentidos }\end{array}$ \\
\hline Parodia & $\begin{array}{l}\text { de la feminidad por usos de la lengua hiperbólicos } \\
\text { y exclamativos, uso vocativos convencionales, uso } \\
\text { del francés como rasgo de amaneramiento }\end{array}$ \\
\hline
\end{tabular}

Fuente: Elaboración y traducción propias.

Como explica claramente, Dimitri Asimakoulas la propuesta de Harvey surge de otra conceptualización previa desarrollada por Jack Babuscio.

\begin{abstract}
Building on Babuscio, Harvey slightly modified the four semiotic strategies of camp [irony, aestheticism, theatricality, humor], extending them to verbal usage. [...] Furthermore his construal of camp addresses a weakness of Babuscio's model, i.e., the fact that it describes the effects of camp and not 'the actual triggers for sing-making/manipulation that underpins them'. (Asimakoulas 58)
\end{abstract}

Además de esto, Harvey plantea que es necesario tomar en cuenta el contexto en el que se enuncia el camp talk para acceder a sus componentes semióticos, pragmáticos y políticos (Harvey, "Translating Camp Talk: Gay Identities and Cultural Transfer" 455). Sin embargo, son estos componentes los que resultan poco accesibles, debido a la dimensión cultural aprendida del camp. Las evidencias microtextuales permiten identificar las características externas, pero muchas veces no son suficientes para interpretarlas como camp, debido a que solo lo locucionario no puede revelar la intención de los interlocutores y, por lo tanto, las evidencias léxicas o microtextuales, en general, no son suficientes para dar cuenta del camp talk. 


\section{El caso de RuPaul's Drag Race y su subtitulación}

Del video musical «Love Shack» (1989) de la banda estadounidense The B-52's, se recuerda mucho una escena en la que Fred Schneider exclama «You are a what?!» a uno de los invitados que se encontraba bailando en la cabaña cerca de la autopista de Atlanta. Tras una brevísima pausa, la música continúa y todos siguen festejando. El personaje interpelado en el video no es otro que RuPaul Charles o RuPaul, a secas, una de las más reconocidas drag queens a nivel mundial, que entonces estaba a punto de hacerse conocido en los medios de comunicación masivos. RuPaul's Drag Race puede considerarse una evidencia del giro LGBTI que ha dado la televisión de Estados Unidos desde finales del siglo xx, un giro cuyos inicios podrían rastrearse hasta otras series de televisión de realidad como Queer Eye for the Straight Guy o comedias de situación como Will \& Grace (Valdeón).

Sin embargo, RPDR también es un hito particular de la cadena de representaciones de las identidades transgénero en los medios de comunicación. En efecto, podría afirmar que la posibilidad de tener este tipo de contenidos en la pantalla chica ha tenido un desarrollo paralelo al que menciona Pérez L. Heredia con respecto a las nuevas representaciones de las mujeres a partir del 2001. El dragqueenismo, por su parte, se ha consolidado como una práctica cultural e identitaria en las comunidades gais, tanto en espacios anglófonos como en países de habla hispana. La migración de esta práctica cultural ha conllevado también contacto lingüístico; la palabra drag queen, por ejemplo, ha resistido los tamices lingüísticos del español. Ha podido desplazarse al castellano mediante un préstamo puro y ha traído consigo un bagaje de experiencias que no han resultado fácilmente transferibles. En efecto, surgen muchos problemas conceptuales cuando se intenta relacionar drag queen con otras palabras como transformista o travesti, debido a las características propias de las identidades de género y sexuales de cada espacio receptor del dragqueenismo (Villanueva Jordán 114). 
Los episodios de RPDR se emiten desde 2009 mediante distintos canales de televisión de pago o transmisión por internet en Estados Unidos y la mayor parte Hispanoamérica. Hasta 2016, la productora World of Wonder ha preparado ocho temporadas de RPDR para la cadena de televisión Logo, además de varios programas y microprogramas derivados para televisión y para su canal de YouTube. Como muchos programas de televisión de realidad, $R P D R$ es una competencia (race); en este caso, los competidores (doce o catorce) buscan que las coronen como la próxima súper estrella del drag. La edición de cada episodio reúne distintos momentos de la convivencia de los participantes, los testimonios individuales, la manera en que se preparan para los retos, los retos propiamente dichos, el juzgamiento y las eliminatorias. Los episodios cuentan así con una secuenciación poco variable a lo largo de las temporadas. Sin embargo, como propondré más adelante, el elemento de cohesión de todos los episodios es el camp talk.

\subsection{Comunidades de seguidores de RPDR en Facebook}

Las plataformas web han sido un espacio de contacto entre personas con intereses similares, desde la aparición de los foros de discusión, pasando por los grupos de blogueros, hasta llegar a los usuarios de Facebook que se reúnen en grupos abiertos, cerrados o secretos, o que siguen las noticias de una página que «les gusta». En el caso de los seguidores de $R P D R$, se pueden encontrar foros de discusión en español relacionados con el programa de televisión, a pesar de que los grupos de seguidores y páginas de Facebook reúnen a miles de seguidores y centralizan la transmisión de información mediante discusiones, votaciones, publicación de noticias, entre otras actividades. Debido a este dinamismo en Facebook, resulta relevante enfocarse en algunas páginas y grupos de seguidores que cuentan con un alto número de seguidores y con una actividad constante. Aunque la información que presento no proviene de un «trabajo etnográfico en línea» ni de una participación constante en estas páginas o grupos, sí soy un lector de la información que 
los administradores y otros miembros de los grupos publican, en muchos casos, diariamente.

Las páginas y grupos de Facebook relacionadas con RPDR y que reúnen a hispanohablantes superan la veintena y esta cantidad puede incrementarse o reducirse con el tiempo. He identificado cuatro páginas y dos grupos de Facebook que cuentan con más de dos mil seguidores y que han mantenido una actividad constante hasta mayo de 2015. A continuación presento algunos datos de estas comunidades de seguidores de Facebook.

Tabla 2 - Comunidades de seguidores de RPDR en Facebook ${ }^{1}$

\begin{tabular}{l|l|l}
\hline Nombre & Tipo & $\begin{array}{l}\text { Cantidad de seguidores } \\
\text { (octubre 2016) }\end{array}$ \\
\hline $\begin{array}{l}\text { RuPaul's Drag Race } \\
\text { (RPDR) en Español }\end{array}$ & Página & $\begin{array}{l}\text { A } 15053 \text { personas les gusta } \\
\text { la página }\end{array}$ \\
\hline $\begin{array}{l}\text { RuPaul's Drag Race } \\
\text { Venezuela }\end{array}$ & Página & $\begin{array}{l}\text { A } 3055 \text { personas les gusta } \\
\text { la página }\end{array}$ \\
\hline $\begin{array}{l}\text { RuPaul's Drag Race } \\
\text { Perú }\end{array}$ & Página & $\begin{array}{l}\text { A } 2141 \text { personas les gusta } \\
\text { esta página }\end{array}$ \\
\hline $\begin{array}{l}\text { RuPaul's Drag Race } \\
\text { Colombia }\end{array}$ & Página & $\begin{array}{l}\text { A } 2050 \text { personas les gusta } \\
\text { esta página }\end{array}$ \\
\hline $\begin{array}{l}\text { RuPaul's Drag Race } \\
\text { México }\end{array}$ & $\begin{array}{l}\text { Grupo } \\
\text { (cerrado) }\end{array}$ & 2637 miembros \\
\hline $\begin{array}{l}\text { RuPaul's Drag Race } \\
\text { Chile }\end{array}$ & $\begin{array}{l}\text { Grupo } \\
\text { (cerrado) }\end{array}$ & 2346 miembros \\
\hline
\end{tabular}

Fuente: Elaboración y traducción propias.

Tal vez sea necesario partir del concepto de comunidades para profundizar en los casos de Facebook. En una importante

\footnotetext{
${ }^{1}$ En el caso de los grupos cerrados, se requiere de la aprobación de un administrador o de un miembro para que el usuario interesado en el grupo pase a ser miembro. Para octubre de 2016, la cantidad de miembros y seguidores se había triplicado en todos los casos, en relación con el primer vaciado de datos en mayo de 2015.
}

Cad. Trad., Florianópolis, v. 39, no 3, p. 156-188, set-dez, 2019. 
contribución sobre los aspectos sociológicos y antropológicos (y de las industrias culturales) de la traducción, Hu definía las affective translation communities como aquellos grupos de personas en los que "the collective act of translation mobilizes resources from around the world in order to sustain emotional investment necesarry for fandom in the absence of traditional advertising and publicity" (Hu 37). Si bien existen otras denominaciones que utilizan, por ejemplo, Jiménez-Crespo (62), non-solicited model, o Kelly, Ray y DePalma (89), cause-driven community translation, el componente afectivo de la propuesta de $\mathrm{Hu}$ (40) pone de relieve la relación emocional entre los miembros del grupo y la práctica traductora procedía, que a su vez reviste los productos traducidos y sirve de recompensa para los traductores.

En ese sentido, no todas las comunidades hispanohablantes de Facebook relacionadas con RPDR pueden considerarse comunidades de traducción afectivas, debido a que la traducción no es un elemento clave en las dinámicas de los grupos. Por ejemplo, en el caso de la página $R P D R$ en Español, los administradores presentan de la página de la siguiente manera.

Somos el Fan Page en español de RuPaul's Drag Race, creado POR FANÁTICOS PARA FANÁTICOS. [...] Página de Fans dedicado a todos los seguidores de habla hispana de RuPaul's Drag Race [...] Esta comunidad pretende ser una plataforma de debate, conversación, cariño y tolerancia, donde también podrás compartir y enterarte sobre las últimas novedades del programa.

Si bien se explicita que la página tendrá contenido en español, no se menciona de forma directa la traducción de contenidos; se privilegian más bien las noticias y las discusiones en español, y se plantea claramente las normas de conducta del grupo (cariño y tolerancia). El componente afectivo es, al parecer, parte de las distintas comunidades de fanáticos, debido a las dinámicas estructurales en la que los seguidores o miembros intercambian bienes culturales, simbólicos, sociales. La vinculación con la traducción tampoco es relevante en el caso de los grupos $R P D R$ México y $R P D R$ Chile; sin embargo, el componente afectivo de 
ambas comunidades sí es identificable por las distintas prácticas que se han desarrollado en ambos grupos. Por ejemplo, en el caso del grupo de México, los miembros publican fotos suyas como drag queens y esperan la valoración del resto de miembros. En el caso del grupo de Chile, las discusiones y el intercambio de comentarios con respecto a los protagonistas del programa de televisión se relaciona con las dinámicas de afecto que menciona $\mathrm{Hu}$ - no solo se trata de intercambios de información racionales, sino de la satisfacción de hablar de un tema, defender una posición a partir del buen gusto y demostrar qué se sabe-.

Las páginas $R P D R$ Colombia, $R P D R$ Perú y $R P D R$ Venezuela mantienen la relación afectiva de las otras tres comunidades; sin embargo, en estas se halla el componente traslativo. En el caso de la página de Venezuela, los administradores la presentan de la siguiente manera: «Una Página Donde Podrás Disfrutar Los Episodios Subtitulado De Las ( $\mathrm{sic}$ ) Nueva Temporada De RuPaul's Drag Race En Logo Tv» (RPDR Venezuela). El subtitulado que mencionan es un elemento central de esta comunidad, debido a que muchos de los seguidores no solo esperan, sino que reclaman -mediante comentarios - los episodios subtitulados en español poco después de su emisión original. De esta manera, esta página publica - 0 , si se prefiere, provee - los episodios subtitulados para los seguidores que, de otra forma, no tendrían acceso por no contar con televisión por cable o bajo demanda, no conocer las plataformas de descarga o retransmisión (ilegales), y principalmente por no conocer la lengua en la que se emite el programa.

La página $R P D R$ Venezuela cuenta con administradores y otros colaboradores que subtitulan, editan y publican los episodios en internet. Se realizan convocatorias un par de veces al año para la conformación de este equipo; sin embargo, en conversación con los administradores de esta página, supe que en 2015 trabajaron solo dos personas para la subtitulación de toda la séptima temporada y preveían que sucedería lo mismo en 2016 (Cadenas Prieto). En el caso de RPDR Colombia, los administradores dicen que se trata de un equipo de cuatro personas que dividen semanalmente cada 
episodio en segmentos de diez minutos para poder publicar los episodios rápidamente (Cataño et al.). En otros casos, un solo seguidor de la página se encarga de la subtitulación de un conjunto de episodios o de una temporada (Reygaga Drag Race).

$R P D R$ Venezuela ha tenido una producción de subtítulos constante a lo largo de los últimos tres años, con la subtitulación de la sexta, séptima y octava temporada (en 2014, 2015 y 2016, respectivamente). Se trata de un ejercicio persistente frente a las distintas ocasiones en las que los repositorios de los videos (canales de YouTube) han sido cancelados por cuestiones de derechos de autor: deben mudar constantemente sus videos y volver a cargarlos para que los seguidores puedan verlos. En el marco de las dinámicas afectivas de estas comunidades que se articulan debido a la producción y consumo de traducciones, la resiliencia de los productores es una característica ineludible.

\subsection{Método de análisis de dos episodios de la sexta temporada de $R P D R$}

Por más que su denominación común sea televisión de realidad, la manera en que estos programas se editan resulta en narrativas contenidas en cada episodio y que se extienden por toda la temporada; ello permite que los televidentes se interesen por seguir el programa cada semana. A pesar de que no existen roles explícitos de buenos y malos, al inicio de cada temporada de $R P D R$, los concursantes asumen posiciones que los muestran como los más hábiles o conocedores, los más críticos, los más débiles. Una característica que todos los participantes deben demostrar desde su entrada en el primer episodio es la capacidad para utilizar el camp talk con agilidad y pertinencia.

Drag queens have a knack for their abillity to play with language, creating inside jokes, catchphrases, and neologisms. Through this bending - or dragging - of language, drag queens create their own vocabulary, one that sets them 
apart from mainstream English language users. This ability for drag queens (and their supporters and fans) to communicate within this particular discourse community and outside perceived language norms is encouraged and celebrated. (Libby 52)

Este hecho motivó que eligiera dos episodios del inicio de la sexta temporada: "RuPaul's Big Opening” y "RuPaul's Big Opening, Part 2". Al ser un inicio de temporada atípico en relación con las temporadas anteriores, la presentación de los trece concursantes se dividió en ambos episodios. Esto permitió que cada participante tuviera suficiente tiempo en pantalla y demostrara sus capacidades lingüísticas antes de ser juzgado en las eliminatorias de fin de episodio.

La sexta temporada es una de las que más éxito de sintonía ha tenido y es la primera temporada que RPDR Venezuela subtituló en su totalidad en 2014. Por su parte, Netflix comenzó a ofrecer el programa de televisión a partir de 2015 y, entonces, su paquete contaba ya con la sexta temporada subtitulada. Tuve acceso a la versión de Netflix con una subscripción regular mientras que, en el caso de la versión de RPDR Venezuela, tuve que encontrar la página web que permitía la descarga directa de los archivos en formato AVI. Realicé una primera transcripción de los subtítulos intralingüísticos (closed captions) de la versión de Netflix, que tomé como referencia para la alineación con los subtítulos interlingüísticos. Luego realicé la transcripción y alineación de las dos versiones subtituladas en español. Cada segmento contó con un código numérico y del concursante; se debe tomar en cuenta que en el primer episodio hubo hasta diez interlocutores, mientras que en el segundo, hasta dieciséis.

La cantidad total de subtítulos intralingüísticos en el caso del primera capítulo fue de 574, mientras que en el caso del segundo episodio fueron 554 subtítulos - 1128 subtítulos por cada una de las tres versiones-. Si comprendemos que el universo textual para este estudio eran los episodios de todas las siete temporadas de $R P D R$ 
que habían sido emitidas hasta el inicio de esta investigación, el corpus específico lo componían los 3384 segmentos. El criterio del muestreo fue la exhaustividad, es decir, analizamos contrastivamente todos los segmentos de las tres versiones. Los criterios de contraste derivaron de una pregunta general exploratoria: ¿qué sucede con el camp talk en la subtitulación al español de RPDR? Asimismo, se consideraron algunas preguntas específicas, aunque también fueron exploratorias. ¿Qué rasgos diferenciales tiene el camp talk en la narrativa escrita en relación con el de los productos audiovisuales? ¿En qué medida los rasgos del camp talk se mantienen en la versión subtitulada de Netflix/de los aficionados?

Para identificar el camp talk en los distintos diálogos de RPDR, se elaboró una lista de ocho rasgos microtextuales (véase el anejo 1) a partir de una primera descripción de ochenta segmentos. La prevalencia de rasgos camp a lo largo de la revisión de los subtítulos permitió establecer categorías a partir de rasgos formales a diferencia de los casos analizados por Harvey ("Camp Talk and Citationality: A Queer Take on 'Authentic' and 'Represented' Utterance"), en los que se trataba de representaciones aisladas cuyas implicancias retóricas debían oponerse al resto del contenido de las novelas para comprenderlas como camp. Los rasgos microtextuales conllevaron una primera etapa de etiquetado de los subtítulos en inglés. Luego se contrastaron los subtítulos de Netflix y los de RPDR Venezuela con los segmentos ya etiquetados para clasificar las traducciones de acuerdo a una lista de diez técnicas (véase el anejo 2) también preparada después de la revisión de los primeros ochenta subtítulos.

Como se mencionó líneas arriba, se evidenció una prevalencia de rasgos camp a medida que se analizaron los subtítulos intralingüísticos; $42 \%$ de los subtítulos fueron etiquetados debido a la presencia de alguna de estas características. Un porcentaje tan alto de este uso lingüístico puede vincularse con sus protagonistas y el hecho de que $R P D R$ busque una representación auténtica de las drag queens: «Stereotypes in drag are nothing new, and stereotypes rearding linguistic patterns of speech are especially common in 
drag communities» (Libby 50). Los usos actuales del camp talk evidenciados en el programa de televisión mantienen los rasgos planteados por autores como Barret o Harvey ("Describing Camp Talk: Language/pragmatics/politics”); de ahí que el repertorio de evidencias del camp de este estudio coincidiera en varios casos con las descripciones que estos autores hicieron en su momento.

Debido a que distintos segmentos de un solo subtítulo podían presentar rasgos camp variados, la cantidad de segmentos etiquetados fue de 590. De estos segmentos, un $43 \%$ recurrían a feminizaciones con base en el uso de distintas categorías gramaticales, con declinación de género o significado relacionado con la feminidad, para referirse a los participantes hombres. Un $22 \%$ fueron creaciones discursivas ingeniosas, que en principio tenían significados o matices sexuales; por otro lado, los juegos de palabras alcanzaron un $7 \%$. El uso de elementos semióticos, en particular, los referentes culturales gais, también fue de un $7 \%$. El uso de interjecciones comúnmente relacionadas con la comunidad de drag queens tuvo un $6 \%$. Tanto el uso de referencias sexuales explícitas como el de palabras que han adquirido un significado distinto en el campo de uso de las drag queens fue de un $5 \%$ cada uno. El uso de insultos relacionados con la identidad de género, características físicas o por cuestiones sexuales alcanzó también un $5 \%$.

\subsubsection{Resultados porcentuales de la subtitulación de Netflix y de RPDR Venezuela}

Las técnicas de traducción propuestas para el análisis contrastivo pueden ser agrupadas entre aquellas que contribuyeron a mantener los rasgos del camp talk (la traducción literal, la feminización, la amplificación, el préstamo, la sustitución, la adición y el calco) y las que eliden estos rasgos (la masculinización, la supresión y la neutralización). En relación con el número total de segmentos con rasgos camp, un $79 \%$ (466) de las técnicas de la subtitulación de Netflix contribuía a mantener estos rasgos; en el caso de la 
subtitulación de $R P D R$ Venezuela, un $82 \%$ (483) contribuía a mantener el camp talk. A pesar de la similitud de los porcentajes, existen claras diferencias entre el uso de técnicas entre ambas versiones subtituladas; estás se mencionan en el siguiente párrafo.

La traducción literal es la más común en la versión de Netflix, al haberse utilizado el $62 \%$ de oportunidades. En el caso de $R P D R$ Venezuela, esta técnica representa un $27 \%$ de las técnicas utilizadas. En la versión subtitulada por aficionados, la técnica más frecuente resulta ser el calco (31 \%) -en particular en relación con las creaciones discursivas y a pesar de que la agramaticalidad y la falta de coherencia de los subtítulos está presente en gran parte de estos segmentos traducidos-. En el caso de Netflix, hubo un $4 \%$ de usos de calco. Este fue el hallazgo más llamativo dado que los repertorios interpretativos del camp incluyen los objetos y prácticas culturales que, por lo regular, provienen de espacios anglófonos: la música pop, las películas de la época dorada de Hollywood, las obras de teatro de Broadway, las series de televisión, los cantantes y otros artistas de fama mundial, el imaginario social (Crimmins 130). Era lógico esperar hallazgos vinculados con modulaciones o adaptaciones derivadas de las restricciones culturales; no fue así.

En relación con lo anterior, se puede mencionar que el uso de préstamos es más común en la versión de RPDR Venezuela (16 \%) en relación con la versión de Netflix (2\%). Asimismo, El uso de amplificaciones para referirse a algunos referentes culturales o juegos de palabras fue de $2 \%$ en ambas versiones. Un rasgo propio de la versión de $R P D R$ Venezuela es la sustitución de un elemento semiótico camp por otro; sucedió en 6 oportunidades, que equivalen a un $1 \%$. Asimismo, se registraron 4 casos de adición en los subtítulos de $R P D R$ Venezuela, equivalentes a un $1 \%$; no hubo adiciones en el caso de Netflix. Interpreto estos casos de sustitución y de adición en $R P D R$ Venezuela como dos evidencias de las funciones sociales del fansubbing: la afectiva que, al surgir a partir del vínculo entre el sujeto y el producto cultural, conlleva que el sujeto prosumidor intervenga (se proyecte a sí mismo) en el texto audiovisual; y la función estética, 
vinculada con la visibilidad de prácticas y productos culturales a veces restringidos en el sistema receptor.

La traducción en femenino alcanzó un $32 \%$ en la versión de Netflix y $24 \%$ en la versión de RPDR Venezuela; al respecto de esta técnica, hubo casos en los que un segmento de la versión original contaba en su traducción con más rasgos camp debido al uso de palabras marcadas en femenino en castellano. En relación con esta técnica, cerca de $58 \%$ de subtítulos intralingüísticos de los dos episodios analizados se etiquetaron como neutrales, debido a que no tenían rasgos camp identificables en inglés. Resulta interesante ver que, tanto en el caso de los subtítulos de Netflix como en los de $R P D R$ Venezuela, se utilizaron categorías gramaticales con marcas de género morfológicas o semánticas que contribuían a mantener el rasgo del camp talk. En el caso de Netflix, hay 55 subtítulos con el género femenino marcado; en el caso de $R P D R$ Venezuela, hay 48 subtítulos en castellano en los que se utilizó la feminización a pesar de que en los subtítulos intralingüísticos no había marcas de género. Estas decisiones, en ambas versiones de los subtítulos en castellano, parecen deberse a las cuestiones de coherencia en las decisiones de los traductores.

\section{Discusión sobre el estudio}

El valor del camp como una categoría de estudio se basa en sus vertientes culturales, particularmente, en las actitudes de los hombres gais afeminados, las perfomances de la drag queen y todos los elementos culturales (del cine, la música o la televisión) que suelen citarse mediante estas expresiones identitarias. El camp no se restringe a los sujetos homosexuales, dado que no se trata de un elemento esencialista que determina, por ejemplo, una identidad sexual. Sin embargo, la recurrencia de estas expresiones en determinadas comunidades de práctica permite la selección de algunas poblaciones para su estudio - lo que se vincularía con el concepto de la sociolingüística denominado indexicality-. Por 
ejemplo, en el caso de las drag queens estadounidenses, el camp llega a constituir gran parte su performance y puede identificarse en el propio nombre de la drag queen, su forma de vestir, las canciones de las que hace fonomímica y la forma en que actúa frente a cada situación: cuando busca hacer reír, insultar, responder a un insulto, entre otras. Estas son algunas características que Rusty Barret consideró durante su estudio sobre drag queens afroamericanas en Tejas (Estados Unidos):

The man in his study used language that was indexical of stereotypical white femininity, black masculinity and homosexuality, a combination of which was used to perform and entirely new drag persona. (Jones 215)

Si bien el postulado base desde el que partí para realizar el estudio contemplaba que, para consumir el camp, se requiere de un capital cultural en constante movilización y que no es accesible a todos los públicos (sean anglófonos o no), la edición del programa permite comprender lo que los concursantes hacen y dicen: mediante la construcción de una narrativa y situaciones comunes en toda la temporada, a las que contribuyen una serie de personajes tipo, sus comentarios fuera de escena, e incluso la musicalización. La accesibilidad del camp talk no depende necesariamente del capital cultural con el que cuente el destinatario, sino de comprender las pautas facilitadas mediante cada episodio, en las temporadas. Quedaría por comprobar si el grado de comprensibilidad era el mismo en temporadas anteriores a la sexta, cuando el programa aún no se había masificado.

A lo anterior podría agregar que el camp, como una estética o sensibilidad, ha calado progresivamente en distintos productos culturales y dinámicas sociales no necesariamente anglófonas. Ello podría deberse, en particular, a que los principales productos que conllevan la aculturación (Crimmins; Halperin) se movilizan en el circuito cultural de masas en el que la comunicación intercultural 
depende de procesos de distribución interlingüísticos. Esto quiere decir que la posproducción de los productos que cumplen una función social en la cultura gay mainstream en inglés recurre a la traducción para continuar su proceso regular de difusión. La subtitulación de $R P D R$ al castellano, ya sea por profesionales o por aficionados, vendría a ser un caso de dicho proceso de difusión cultural.

A partir del contraste con los subtítulos de Netflix y RPDR Venezuela, podría decirse que ambas versiones mantienen la mayoría de rasgos del camp talk; solo un $18 \%$ (RPDR Venezuela) y 21 (Netflix) de los segmentos traducidos han perdido los rasgos identificados en la versión en inglés. Los insultos, algunos referentes culturales y los juegos de palabras, en principio, serían los rasgos elididos en ambos casos. Las técnicas de Netflix para traducir literalmente todos los diálogos y enunciados de los participantes sería una consecuencia, precisamente, de que el camp talk está facilitado mediante la propia edición del programa y de procesos de aculturación previos a la propia difusión de este programa. En el caso de RPDR Venezuela, la técnica más utilizada vendría a ser el calco. Como se mencionó antes, en estos casos se pudo identificar varios errores de sentido y segmentos incomprensibles, resultado de construcciones agramaticales. Las observaciones sobre la calidad de los subtítulos por aficionados que hicieran Díaz Cintas y Muñoz Sánchez, O’Hagan y Secara son aplicables también en este caso.

En los dos casos de las versiones en castellano, se aprecia coherencia en el uso del femenino en distintos rasgos microtextuales a lo largo de los dos episodios analizados, tanto en los segmentos marcados como camp y aquellos que se consideraron neutrales en la versión en inglés. La coherencia de otras decisiones en el caso de Netflix puede notarse en que no usan préstamos del inglés, como sí sucede en la versión de RPDR Venezuela. El uso de palabras del camp talk en castellano por parte determinados grupos sociales (identitarios) en Sudamérica podría explorarse debido al creciente consumo de este programa de televisión de realidad. Quedaría por explorar, entonces, si los productos culturales traducidos pueden promover la transculturación de determinados códigos culturales 
con todo lo que ello implica en el ámbito de la subjetividad de los sujetos consumidores.

Este último planteamiento no es nuevo en el campo de la traductología, como tampoco lo es el interés por los estudios de género, los estudios gais o la teoría queer en la traducción. Sin embargo, queda pendiente determinar en qué medida estos elementos culturales gais implican restricciones para los destinatarios hispanófonos. Hay argumentos que proponen que los referentes culturales arraigados en una cultura impiden que el camp desencadene sus efectos (Asimakoulas 68), mientras que otros proponen que los modelos de la identidad gay se reconocen regularmente en espacios heterosexuales (Valdeón 89). Sin embargo, ambas posturas provienen de espacios receptores del camp diferenciados (Grecia y España, respectivamente). Es posible que las restricciones y la recepción del camp sean distintas en Latinoamérica, en cada país y en cada grupo social que consuma algún producto cultural con estos componentes.

En relación con la práctica de la subtitulación por aficionados, antes puse de relieve el componente traslativo y afectivo del caso de RPDR Venezuela. Sin embargo, su propuesta también abarca aspectos estéticos y políticos. Para ambos administradores de esta página, la subtitulación de $R P D R$ apunta a difundir "el arte de ponerse en drag, de maquillarse con mucho detalle, de bailar y cómo se puede hacer del arte un modo de vida" (Reygaga Drag Race). Para ellos, mejorar la concepción del dragqueenismo implica, a su vez,

menos discriminación de la población LGBT, las personas trans particularmente, porque en RPDR ya ha habido mujeres trans; cuando subtitulamos visibilizamos más nuestra cultura. (Cadenas Prieto)

En ese sentido, se trataría de un caso en el que la subtitulación por aficionados reúne hasta cinco funciones sociales: la función 
traslativa (de accesibilidad a contenidos en lenguas extranjeras), la comunitaria (que permite reunir a sujetos en comunidades de práctica), la estética (que permite la circulación de productos culturales y el establecimeinto de poéticas), la afectiva (que conlleva el vínculo subjetivo entre prosumidores y productos) y la política (relacionada con la promoción de significados alternativos a los aceptados socialmente).

Este tipo de subtítulos no se asemejan a las características usuales de los fansubs descritos en Díaz Cintas y Muñoz Sánchez, sino que se parecen más a las propuestas de ViKi y el "perfil profesional" que se busca alcanzar (Dwyer). Con respecto a la calidad, la subtitulación es legible porque se trabaja con programas digitales, de acuerdo a una suerte de protocolos implícitos sobre la duración, tipografía y extensión de los subtítulos; sin embargo, su inteligibilidad y su coherencia son cuestionables, debido a que no cuentan con pautas claras para la revisión de los productos. En el caso de $R P D R$ Venezuela, ambos administradores han dividido las tareas de subtitulación de acuerdo a sus habilidades; por ello, la traducción de los diálogos está a cargo de uno de ellos, mientras que la edición de los subtítulos (con MS ${ }^{\circledR}$ Windows Movie Maker ${ }^{\circledR}$ ) está a cargo del otro. Sin embargo, al tratarse de una actividad compartida, ambos han mejorado sus competencias en estos campos y ahora ambos pueden controlar la calidad de los subtítulos y buscar freeware para que, por ejemplo, las imágenes no pierdan calidad después de la incrustación de los subtítulos.

Como mencioné antes, a veces la comprensión del texto fuente es mínima y se encuentran estructuras calcadas o segmentos sin traducir. Sin embargo, estas decisiones no pueden proceder necesariamente del desconocimiento, sino también de criterios de agencia y estéticos que determinan el producto final; es decir, los segmentos que son calcados podrían derivar del interés de los subtituladores por mantener algún rasgo de extrañeza en los textos meta como se ha documentado extensamente en otros estudios.

Por otro lado, las características estéticas o normas operacionales de los procesos de subtitulación de RPDR Venezuela obedecen 
o resultan del conocimiento general y autoformación de los traductores aficionados, avanzan a un ritmo diferenciado en cada grupo subtitulador y no cuentan con un proceso establecido, en el que las tareas estén claramente divididas y que también está condicionado por la escasez de recursos humanos (colaboradores). En el caso de RPDR Venezuela fueron solo dos personas las encargadas de subtitular toda una temporada sin utilizar freeware de subtitulación, sino solo el programa Windows Movie Maker. Solo a partir de la temporada 8 de 2016, ya avisaban a sus seguidores que comenzarían a trabajar con subtítulos SRT. Las cuestiones de aseguramiento de la calidad de los productos por aficionados o de la traducción comunitaria parecen ser cuestiones ajenas aún en estas comunidades de aficionados.

Al momento de redactar esta sección del artículo, la página RPDR Venezuela había dejado de publicar episodios subtitulados de la octava temporada. Por su parte, la página RPDR Colombia había tomado la posta desde febrero de 2016; se mantuvo actualizada hasta finales de octubre con la subtitulación de la temporada All Stars 2 y con mensajes semanales que aseguraban a los seguidores que tendrían los episodios subtitulados al día siguiente de su emisión. A partir de estos casos, las funciones sociales de la subtitulación parecen poner de relieve el componente afectivo de esta práctica traductora que, a su vez, conduce a la conformación de comunidades de práctica, no solo vinculados por el programa de televisión, sino también por la traducción o los productos traducidos (Pérez-González). 


\section{Referencias}

Asimakoulas, Dimitri. "Dude Looks like a Lady. Hijacking Transsexual Identity in the Subtitled Version of Strella by Panos Koutras". The Translator 18.1 (2012): 47-75.

Barret, Rusty. "Supermodels of the World, Unite! Political Economy and the Language of Performance among African American Drag Queens”. The Language and Sexuality Reader. London: Routledge, 2006.

Bergman, David. "Strategic Camp: The Art of Gay Rhethoric". Camp Grounds. Style and Homosexuality. Amherst: University of Massachusets Press, 1993.

Booth, Mark. "Campe-Toi! On the Origins and Definitions of Camp". Camp. Queer Aesthetics and the Performing Subject: A Reader. Ann Arbor: The University of Michigan Press, 2000.

Cadenas Prieto, Londres. Entrevista a Administradores de La Página de Facebook RuPaul's Drag Race Venezuela. 2016.

Cameron, Deborah, and Kulick, Don. Language and Sexuality. Cambridge: Cambridge University Press, 2003.

Cataño, Diego et al. Entrevista Con Diego Cataño, Lisseth Gómez Y Caleb Green, Administradores de La Página de Facebook RuPau'ls Drag Race Colombia. 2016.

Crimmins, Cathy. Los Homosexuales Al Rescate de La Civilización. Una Historia Verdadera Y Heroica de Cómo Los Gays Salvaron El Mundo Moderno. Trans. Alberto Mira. Barcelona: Egales, 2004.

Díaz Cintas, Jorge and Pablo Muñoz Sánchez. "Fansubs: Audiovisual Translation in an Amateur Environment". JoSTrans - The Journal of Specialised Translation 6 (2006): 37-52. 
Dwyer, Tessa. "Fansub Dreaming on ViKi 'Don't Just Watch But Help When You Are Free'". The Translator 18.2 (2012): 217-43. < https://doi.org/10.1080/ 13556509.2012.10799509>

Gray, John. "Language and Non-Normative Sexual Identities". In: Preece, Siân. (Ed). The Routledge Handbook of Language and Identity. London: Routledge, 2016.

Hall, Stuart. Representation: Cultural Representations and Signifying Practices. Thousand Oaks: Sage Publications, 1997.

Halperin, David. How to Be Gay. Cambridge: Harvard University Press, 2012.

Harvey, Keith. "Camp Talk and Citationality: A Queer Take on 'Authentic' and 'Represented' Utterance”. Journal of Pragmatics 34 (2002): 1145-65.

Harvey, Keith. "Describing Camp Talk: Language/pragmatics/politics". Language and Literature 9.3 (2000): 240-60.

Harvey, Keith. "Gay Community, Gay Identity and the Translated Text". TTR: Traduction, Terminologie, Rédaction 13.1 (2000): 137-65.

Harvey, Keith. "Translating Camp Talk: Gay Identities and Cultural Transfer". The Translation Studies Reader. London: Routledge, 2000.

Hayes, Joseph. "Gayspeak." The Language and Sexuality Reader. London: Routledge, 1981.

$\mathrm{Hu}$, Brian. "Korean TV Serials in the English-Language Diaspora: Translating Difference Online and Making It Racial”. The Velvet Light Trap 66 (2010): 36-49.

Jiménez-Crespo, Miguel. "Collaborative and Volunteer Translation and Interpreting”. Researching Translation and Interpreting. London: Routledge, 2016.

Jones, Lucy. "Language and Gender Identities". The Routledge Handbook of Language and Identity. Ed. Siân Preece. London: Routledge, 2016. 
Kelly, N. et al. "From Crawling to Sprinting: Community Translation Goes Mainstream". Linguistica Antverpiensia. New Series - Themes in Translation Studies 10 (2011): 75-94. < https://lans-tts.ua.ac.be/index.php/LANS-TTS/ article/view/278>

Legman, Gershon. "The Language of Homosexuality: An American Glossary". The Language and Sexuality Reader. Ed. Deborah Cameron and Don Kulick. London: Routledge, 2006.

Libby, Anthony. "Dragging with an Accent: Linguistic Stereotypes, Language Barriers and Translingualism". In: Daems, Jim. (Ed). The Makeup of Rupaul's Drag Race. Essays on the Queen of Reality Shows. Jefferson: McFarland \& Company, 2014.

Newton, Esther. Mother Camp. Female Impersonators in America. Chicago: The University of Chicago Press, 1972.

O'Hagan, Minako. "Fan Translation Networks: An Accidental Translator Training Environment?". In: Daems, Jim. (Ed). Translator and Interpreter Training. Issues, Methods and Debates. Continuum, 2008.

Paulson, Don, and Roger Simpson. An Evening at the Garden of Allah. A Gay Cabaret in Seattle, New York. New York: Columbia University Press, 1996.

Pérez-González, Luis. Audiovisual Translation: Theories, Methods and Issues. London: Routledge, 2014. < https://www.escholar.manchester.ac.uk/uk-ac-manscw:256644>

Pérez L. Heredia, María. "Translating Gender Stereotypes: An Overview on Global Telefiction”. Otras Modernidades 2 (2016): 166-81.

Ranzato, Irene. "Gayspeak and Gay Subjects in Audiovisual Translation: Strategies in Italian Dubbing”. Meta: Journal des Traducteurs 57.2 (2012): 369. $<$ https://doi. org/10.7202/1013951ar >

Reygaga Drag Race. Entrevista a Administradores de La Página de Facebook RuPaul's Drag Race Venezuela. 2016. 
Secara, Alina. "R U Ready 4 New Subtitles? Investigating the Potential of Social Translation Practices and Creative Spelling". Linguistica Antverpiensia. New Series - Themes in Translation Studies 10 (2011): 153-71.

Sontag, Susan. "Notes on 'Camp'”. Against Interpretation and Other Essays. London: Picador; Macmillan, 1990.

The Adventures of Priscilla, Queen of the Desert. Dir. Stepahn Elliot. Universal City: Gramercy Pictures, 1994. Film.

The Birdcage. Dir. Mike Nichols. Hollywood: United Artists, 1996. Film.

To Wong Foo, Thanks for Everything! Julie Newmar. Dir. Beeban Kidron. New York: Universal Pictures, 1995. Film.

Valdeón, Roberto. "Schemata, Scripts and the Gay Issue in Contemporary Dubbed Sitcoms". Target 22.1 (2010): 71-93.

Villanueva Jordán, Iván. “'Yo Soy Una Drag Queen, No Soy Cualquier loco' Representaciones Del Dragqueenismo En Lima, Perú”. Península 12.2 (2017): 95-118. <https://doi.org/10.1016/j.pnsla.2017.06.005>.

Recebido em: 15/02/2019 Aceito em: 16/07/2019 Publicado em: Setembro de 2019

Iván Alejandro Villanueva. E-mail: ivan.villanueva@upc.pe ORCID: https://orcid.org/0000-0003-1479-1627 


\section{ANEJO 1 - Rasgos microtextuales del camp talk en RPDR}

\begin{tabular}{|c|c|c|}
\hline Denominación y definición & Código & Ejemplo \\
\hline $\begin{array}{l}\text { Interjección. Expresión lexicalizada con cierto } \\
\text { grado de variabilidad en su combinación y sin } \\
\text { funciones gramaticales, cuyo uso manifiesta, } \\
\text { por lo general, exclamaciones, impresiones o } \\
\text { sentimientos. }\end{array}$ & INX & $\begin{array}{l}\text { ¡Yes, mamma! ¡Gurl! } \\
\text { ¡Party! ¡Escandalo! }\end{array}$ \\
\hline $\begin{array}{l}\text { Feminización. Uso de palabras con género } \\
\text { semántico o morfológico femenino, así como } \\
\text { otros marcadores gramaticales de género para } \\
\text { referirse a un sujeto varón. }\end{array}$ & FEM & Oh, no girl... \\
\hline $\begin{array}{l}\text { Insulto. Ofensa mediante el uso de sustantivos } \\
\text { o adjetivos -no necesariamente peyorativos- } \\
\text { que se enfocan en el aspecto físico, las } \\
\text { cuestiones de género o el nivel de desempeño } \\
\text { artístico. }\end{array}$ & SHD & $\begin{array}{l}\text { Basic bitches no } \\
\text { wanted... }\end{array}$ \\
\hline $\begin{array}{l}\text { Referencia sexual. Palabras o enunciados } \\
\text { de mayor extensión que hacen referencia a } \\
\text { prácticas sexuales. }\end{array}$ & SEX & ¡Gah-ahh-ging! \\
\hline $\begin{array}{l}\text { Resemantización. Reducción, ampliación } \\
\text { o cambio del significado de una palabra } \\
\text { extendido en el uso de los interlocutores. }\end{array}$ & RES & $\begin{array}{l}\text { The following preview } \\
\text { has been declared } \\
\text { sickening for all } \\
\text { audiences. }\end{array}$ \\
\hline $\begin{array}{l}\text { Juego de palabras. Empleo ingenioso de un } \\
\text { conjunto de palabras para permitir distintas } \\
\text { interpretaciones a partir de sus aspectos } \\
\text { morfológicos o fonéticos similares. }\end{array}$ & PUN & $\begin{array}{l}\text { Put the fear in fierce. } \\
\text { - There is tears as well } \\
\text { as fears. }\end{array}$ \\
\hline $\begin{array}{l}\text { Elemento semiótico. Empleo de palabras con } \\
\text { un significado distinto al que les corresponde } \\
\text { propiamente, pero con alguna semejanza } \\
\text { o conexión con elementos culturales de la } \\
\text { comunidad. }\end{array}$ & SEM & $\begin{array}{l}\text { Drop dead gorgeous } \\
\text { fashion. }\end{array}$ \\
\hline $\begin{array}{l}\text { Creación discursiva. Formulación o enunciado } \\
\text { ingenioso que hace referencia a las identidades } \\
\text { de género o a temas sexuales. }\end{array}$ & DIS & $\begin{array}{l}\text { All participants need } \\
\text { to show they have } \\
\text { charisma, uniqueness, } \\
\text { nerve, and talent. } \\
\text { [CUNT] }\end{array}$ \\
\hline $\begin{array}{l}\text { Neutral. Segmento que no cuenta con marcas } \\
\text { evidencias de camp talk. }\end{array}$ & NEU & $\begin{array}{l}\text { We started together at } \\
\text { the same club. }\end{array}$ \\
\hline
\end{tabular}

Fuente: Elaboración propia. 


\section{ANEJO 2 - Rasgos microtextuales de la de traducción del camp talk}

\begin{tabular}{|c|c|c|c|}
\hline Denominación y definición & Código & Versión & Ejemplos \\
\hline \multirow{2}{*}{$\begin{array}{l}\text { Feminización. Se mantiene } \\
\text { o agrega una marca de } \\
\text { género gramatical femenino } \\
\text { en relación con los rasgos } \\
\text { del texto fuente. }\end{array}$} & \multirow[t]{2}{*}{ TFE+ } & V.F. & $\begin{array}{l}\text { We started together at the same } \\
\text { club. }\end{array}$ \\
\hline & & RPVEN & $\begin{array}{l}\text { Nosotras empezamos juntas en el } \\
\text { mismo club. }\end{array}$ \\
\hline \multirow{2}{*}{$\begin{array}{l}\text { Masculinización. Se } \\
\text { remplaza un elemento con } \\
\text { marca de género gramatical } \\
\text { femenino por un marcador } \\
\text { masculino. }\end{array}$} & \multirow[t]{2}{*}{ MAS- } & V. F. & $\begin{array}{l}\text { Now remember, if you can't love } \\
\text { yourselves, how in the hell are you } \\
\text { gonna love somebody else? Can I } \\
\text { get an amen up in here? }\end{array}$ \\
\hline & & RPVEN & $\begin{array}{l}\text { Y recuerden, si no puedes amarte a } \\
\text { ti mismo, ¿Cómo diablos amarás a } \\
\text { alguien más? }\end{array}$ \\
\hline \multirow{2}{*}{$\begin{array}{l}\text { Traducción literal. Se } \\
\text { traduce palabra por palabra } \\
\text { un segmento del texto } \\
\text { fuente con la finalidad de } \\
\text { mantener el rasgo camp } \\
\text { sin que haya problemas de } \\
\text { gramaticalidad. }\end{array}$} & \multirow[t]{2}{*}{ LIT+ } & V.F. & $\begin{array}{l}\text { I'm April Carrion. I'm } 24 \text { years } \\
\text { old directly from Guaynabo, Puerto } \\
\text { Rico. }\end{array}$ \\
\hline & & RPNET & $\begin{array}{l}\text { Soy April Carrión. Tengo } 24 \text { años. } \\
\text { Vengo directamente de Guaynabo. } \\
\text { Puerto Rico. }\end{array}$ \\
\hline \multirow[t]{2}{*}{$\begin{array}{l}\text { Calco. Se traduce palabra } \\
\text { por palabra un segmento del } \\
\text { inglés a pesar de su posible } \\
\text { agramaticalidad. }\end{array}$} & \multirow[t]{2}{*}{$\mathrm{CAL}+$} & V.F. & $\begin{array}{l}\text { I'm walking it down that runway } \\
\text { and I'm serving down this thing. } \\
\text { I'm giving you old school style New } \\
\text { York walk, walk, walk, pose. }\end{array}$ \\
\hline & & RPVEN & $\begin{array}{l}\text { Estoy caminando por la pasarela y } \\
\text { estoy sirviendo hacia abajo, Miss } \\
\text { Thing Estoy sirviendo el estilo Old- } \\
\text { School de Nueva York camino, } \\
\text { camino, camino, pose. }\end{array}$ \\
\hline \multirow{2}{*}{$\begin{array}{l}\text { Préstamo. Se incorpora } \\
\text { una palabra del inglés - } \\
\text { que representa un rasgo } \\
\text { camp- sin traducirla o con } \\
\text { algún tipo de adaptación } \\
\text { lingüística - gráfica/fónica/ } \\
\text { morfológica-. }\end{array}$} & \multirow[t]{2}{*}{ PRS + } & V. F. & $\begin{array}{l}\text { Thanks, mama, for being so fishy } \\
\text { with the fish. }\end{array}$ \\
\hline & & RPVEN & $\begin{array}{l}\text { Gracias mama por ser tan Fishy de } \\
\text { lo Fish. }\end{array}$ \\
\hline
\end{tabular}




\begin{tabular}{|c|c|c|c|}
\hline \multirow{2}{*}{$\begin{array}{l}\text { Supresión. Se eliminan } \\
\text { rasgos del camp sin que } \\
\text { el texto meta presente } \\
\text { equivalentes formales } \\
\text { siquiera. }\end{array}$} & \multirow[t]{2}{*}{ SPR- } & V. F. & ¡GAH-AHH-GING! \\
\hline & & RPNET & [No se tradujo.] \\
\hline \multirow{2}{*}{$\begin{array}{l}\text { Neutralización. Se } \\
\text { remplazan rasgos del } \\
\text { camp por otros elementos } \\
\text { carecen de carga pragmática } \\
\text { o semiótica sin ser } \\
\text { compensados. }\end{array}$} & \multirow[t]{2}{*}{ NEU- } & V. F. & Drop dead gorgeous fashion. \\
\hline & & RPNET & $\begin{array}{l}\text { Moda espectacular y } \\
\text { despampanante. }\end{array}$ \\
\hline \multirow{2}{*}{$\begin{array}{l}\text { Adición. Se incluye un } \\
\text { rasgo del camp que no } \\
\text { figuraba en el texto fuente. }\end{array}$} & \multirow[t]{2}{*}{$\mathrm{ADI}+$} & V.F. & We made it back! \\
\hline & & RPVEN & Lo logramos perras. \\
\hline \multirow{2}{*}{$\begin{array}{l}\text { Amplificación. Explicación } \\
\text { o explicitación de un rasgo } \\
\text { del camp talk mediante el } \\
\text { uso de más significantes. }\end{array}$} & \multirow[t]{2}{*}{$\mathrm{AMP}+$} & V. F. & $\begin{array}{l}\text { Kelly mantle, your runaway look } \\
\text { was a little downtown shabby. I'm } \\
\text { sorry but you're up for elimination. }\end{array}$ \\
\hline & & RPVEN & $\begin{array}{l}\text { Shabby: lamentable [Aparece en } \\
\text { la parte superior de la pantalla en } \\
\text { letras blancas] }\end{array}$ \\
\hline \multirow[t]{2}{*}{$\begin{array}{l}\text { Sustitución. Se remplaza un } \\
\text { rasgo camp por otro. }\end{array}$} & \multirow[t]{2}{*}{ SUS+ } & V. F. & $\begin{array}{l}\text { The following preview has } \\
\text { been declared sickening for all } \\
\text { audiences. }\end{array}$ \\
\hline & & RPVEN & $\begin{array}{l}\text { La siguiente VISTA PREVIA ha } \\
\text { sido declarada } F E R O Z \text { para todas } \\
\text { LAS AUDIENCIAS [Mayúsculas } \\
\text { de la versión subtitulada] }\end{array}$ \\
\hline
\end{tabular}

Fuente: Elaboración propia.

* Notas: V.F.: versión fuente; RPNET: subtítulos extraídos de Netflix; RPVEN: subtítulos extraídos de RPDR Venezuela. 